\title{
A Crank-Nicolson linear difference scheme for a BBM equation with a time fractional nonlocal viscous term
}

Xue Shen ${ }^{1}$ and Ailing Zhu ${ }^{1 *}$ (B)

"Correspondence:

zhual@sdnu.edu.cn

'school of Mathematical and

Statistics, Shandong Normal

University, Jinan, China

\section{Springer}

\begin{abstract}
In this paper, we construct a Crank-Nicolson linear finite difference scheme for a Benjamin-Bona-Mahony equation with a time fractional nonlocal viscous term. The stability and convergence of the proposed numerical scheme are rigorously derived. Theoretical analysis shows that the numerical scheme is convergent in the order of $O\left(\tau^{\frac{3}{2}}+h^{2}\right)$, where $\tau$ and $h$ are the time and space step sizes. Two numerical experiments are presented to verify that the theoretical analysis is accurate and to demonstrate that the numerical scheme is effective.
\end{abstract}

MSC: $65 \mathrm{~N} 15 ; 65 \mathrm{~N} 30$

Keywords: BBM equation; Time fractional nonlocal viscous term; Finite difference scheme; Stability; Convergence

\section{Introduction}

In many nonlinear partial differential equations, the most common method to stimulate the nonlinear dispersion wave is by using the Korteweg-de Vries (KdV) equation [1]

$$
u_{t}+u_{x}+u_{x x x}+u u_{x}=0,
$$

because it obeys conservation laws and has been widely applied in solid, liquid, and other subject fields [2]. As an improvement of the KdV equation, generalized regularized longwave (GRLW) equation [3, 4], Rosenau-Burgers (R-B) equation [5, 6], and BenjaminBona-Mahony (BBM) equation [7] can better simulate shallow water waves. Among them, the BBM equation, which describes the unidirectional propagation of surface water in a nonlinear dispersive medium with small amplitude and long wave, is the most typical model [8-13].

Generally, it is difficult to obtain the analytic solution of the aforementioned water wave equation. Therefore, the investigation of using numerical methods to simulate the solutions of the classical water wave equation and the regularized water wave equation has been the subject of many studies in the recent literature (see, for example, [14-20] and the references therein). More recently, Zhang and Xu [10] constructed two linearized schemes for the time fractional water wave equation in a BBM form. Li [21] discussed the linearized difference scheme for a BBM equation with a space fractional nonlocal viscous term.

(c) The Author(s) 2018. This article is distributed under the terms of the Creative Commons Attribution 4.0 International License (http://creativecommons.org/licenses/by/4.0/), which permits unrestricted use, distribution, and reproduction in any medium, provided you give appropriate credit to the original author(s) and the source, provide a link to the Creative Commons license, and indicate if changes were made. 
When simulating the nonlinear differential equation, it is effective to construct the linear difference scheme, which reduces the complexity in computing and the difficulties in the analysis of convergence. Zhang and $\mathrm{Xu}$ [10] used the linearized difference method to solve the nonlinear time fractional water wave equation in a BBM form. However, the different method that is constructed above is a three-layer linear scheme, which does not minimize the amount of calculation. Inspired by the work [21], we find that the Crank-Nicolson method can be combined with the technique of linearization for a nonlinear equation to establish a two-level linear difference scheme, which can further reduce the computational complexity while maintaining the accuracy. Consequently, in this paper, we consider a Crank-Nicolson linear difference scheme for the initial and boundary value problems of the following BBM equation with a time fractional nonlocal viscous term [12]:

$$
u_{t}+u_{x}-\beta u_{x x t}+\epsilon_{0}^{\frac{1}{2} C} D_{t}^{\frac{1}{2}} u+\gamma u u_{x}-\alpha u_{x x}=0, \quad x \in(a, b), t \in(0, T]
$$

and an initial condition

$$
u(x, 0)=u_{0}(x), \quad x \in(a, b)
$$

and the boundary conditions

$$
u(a, t)=u(b, t)=0, \quad t \in(0, T]
$$

where $\beta, \epsilon, \gamma$, and $\alpha$ are positive parameters, ${ }_{0}^{C} D_{t}^{\frac{1}{2}}$ is $\frac{1}{2}$-order Caputo's fractional derivative operator. Ordinarily, the $\alpha$-order Caputo time fractional derivative ${ }_{0}^{C} D_{t}^{\alpha} u(x, t)$ is defined as follows [22]:

$$
{ }_{0}^{C} D_{t}^{\alpha} u(x, t):=\frac{\partial^{\alpha} u(x, t)}{\partial t^{\alpha}}= \begin{cases}\frac{1}{\Gamma(m-\alpha)} \int_{0}^{t}(t-s)^{m-\alpha-1} \frac{\partial^{m} u(x, s)}{\partial s^{m}} d s, & m-1<\alpha<m, \\ \frac{\partial^{m} u(x, t)}{\partial t^{m}}, & \alpha=m \in N,\end{cases}
$$

where $\Gamma(\cdot)$ is a gamma function.

In this paper, we use the $L 1$ formula to approximate $\frac{1}{2}$-order Caputo's fractional derivative; we use the central difference to approximate both the first and second space derivative; we use the linear difference to approximate the nonlinear term; and we use the CrankNicolson difference formula to approximate the first-order time derivatives. Then, we construct a Crank-Nicolson-L1 (C-N-L1) linear difference scheme for problems (2)-(4). The stability and convergence of the $\mathrm{C}-\mathrm{N}-L 1$ numerical scheme are rigorously derived. Numerical experiments show that the theoretical analysis is accurate and that the numerical scheme is effective. The contribution of this paper is to propose, analyze, and verify the combination scheme of a Crank-Nicolson scheme and linearization of nonlinear term.

The outline of this paper is as follows. Section 2 is devoted to the design of the C-N$L 1$ linear difference scheme for the time fractional nonlinear BBM equation. Sections 3 and 4 prove that the scheme is stable and convergent, respectively. Two numerical examples are presented to verify the theoretical result and the effectiveness of the numerical scheme and to demonstrate the impact of various terms by changing the parameters of the corresponding terms in Sect. 5. Finally, the conclusions of this paper are provided. 


\section{The derivation of the C-N-L1 linear difference scheme}

In this section, we describe the derivation of the $\mathrm{C}-\mathrm{N}-L 1$ linear difference scheme for the time fractional BBM equation (2).

We consider the difference scheme for equation (2) in a finite domain $(x, t) \in[a, b] \times$ $[0, T]$. Spatial step size $h=\frac{b-a}{M}$ and time step size $\tau=\frac{T}{N}$, respectively, where $M$ and $N$ are integers and assume that the mesh sizes $h$ and $\tau$ satisfy $0<h<1$ and $0<\tau<1$. Grid points $\left(x_{j}, t_{n}\right)$ are defined by $x_{j}=a+j h(0 \leq j \leq M) ; t_{n}=n \tau(0 \leq n \leq N)$. Let $u_{j}^{n}$ denote the numerical approximation of $u\left(x_{j}, t_{n}\right)$. Sometimes, suppose that $u^{n}=\left\{u_{j}^{n} \mid 0 \leq j \leq M\right\}$ is a grid function. We introduce the following notations and an $L 1$ approximation formula of the $\frac{1}{2}$-order Caputo's fractional derivative [23]:

$$
\begin{aligned}
& u_{j}^{n+\frac{1}{2}}=\frac{u_{j}^{n}+u_{j}^{n+1}}{2}, \quad D_{t} u_{j}^{n}=\frac{u_{j}^{n+1}-u_{j}^{n}}{\tau}, \quad D_{+} u_{j}^{n}=\frac{u_{j+1}^{n}-u_{j}^{n}}{h}, \\
& D_{-} u_{j}^{n}=\frac{u_{j}^{n}-u_{j-1}^{n}}{h}, \quad D^{2} u_{j}^{n}=D_{+} D_{-} u_{j}^{n}=\frac{u_{j+1}^{n}-2 u_{j}^{n}+u_{j-1}^{n}}{h^{2}}, \\
& D_{0} u_{j}^{n}=\frac{u_{j+1}^{n}-u_{j-1}^{n}}{2 h}, \quad\left(u^{n}, v^{n}\right)=\sum_{j=0}^{M-1} h u_{j}^{n} v_{j}^{n}, \quad\left\|u^{n}\right\|=\sqrt{\left(u^{n}, u^{n}\right),} \\
& \left\|u^{n}\right\|_{\infty}=\max _{0 \leq j \leq M-1}\left|u_{j}^{n}\right|, \quad(u, v)_{1}=(u, v)+\beta\left(D_{+} u, D_{+} v\right),
\end{aligned}
$$

and

$$
\frac{1}{\Gamma\left(\frac{1}{2}\right)} \int_{0}^{t_{n}} \frac{u^{\prime}(s)}{\left(t_{n}-s\right)^{\frac{1}{2}}} d s:=\frac{\tau^{-\frac{1}{2}}}{\Gamma\left(\frac{3}{2}\right)}\left(u\left(t_{n}\right)-\sum_{k=1}^{n-1}\left(a_{n-k-1}-a_{n-k}\right) u\left(t_{k}\right)-a_{n-1} u\left(t_{0}\right)\right),
$$

where $a_{j}=(j+1)^{1-\alpha}-j^{1-\alpha}$ and $j \geq 0$.

We use the $L 1$ formula to approximate the Caputo fractional derivatives, use the central differences to approximate for both first and second space derivatives, use the linear differences to approximate the nonlinear term and use the Crank-Nicolson difference formula to approximate the first-order time derivative. Then, we get the C-N-L1 scheme for problems (2)-(4):

$$
\begin{aligned}
& D_{t} u_{j}^{n}+D_{0} u_{j}^{n+\frac{1}{2}}-\beta D_{t} D^{2} u_{j}^{n}+\frac{\epsilon^{\frac{1}{2}} \tau^{-\frac{1}{2}}}{\Gamma\left(\frac{3}{2}\right)}\left(u_{j}^{n+\frac{1}{2}}-\sum_{i=0}^{n-1}\left(a_{i}-a_{i+1}\right) u_{j}^{n-\frac{1}{2}-i}-a_{n} u_{j}^{0}\right) \\
& \quad+\frac{\gamma}{3}\left(u_{j}^{n} D_{0} u_{j}^{n+\frac{1}{2}}+D_{0}\left(u_{j}^{n} u_{j}^{n+\frac{1}{2}}\right)\right)-\alpha D^{2} u_{j}^{n+\frac{1}{2}}=0, \\
& u_{j}^{0}=u_{0}\left(x_{j}\right), \quad 0 \leq j \leq M, \\
& u(a, t)=u(b, t)=0, \quad 0 \leq n \leq N .
\end{aligned}
$$

In order to prove the stability and convergence of the above constructed C-N-L1 scheme, we collect useful facts on the truncation error of the $L 1$ approximate operator for the Caputo fractional derivatives, the discrete Sobolev inequality, and the discrete Gronwall inequality. 
Lemma 2.1 ([24]) Suppose $f(t) \in C^{2}\left[0, t_{n}\right]$. Let

$$
\begin{aligned}
\bar{R}\left(f\left(t_{n}\right)\right):= & \frac{1}{\Gamma(1-\alpha)} \int_{0}^{t_{n}} \frac{f^{\prime}(s)}{\left(t_{n}-s\right)^{\alpha}} d s \\
& -\frac{\tau^{-\alpha}}{\Gamma(2-\alpha)}\left(a_{0} f\left(t_{n}\right)-\sum_{j=1}^{n-1}\left(a_{n-j-1}-a_{n-j}\right) f\left(t_{j}\right)-a_{n-1} f\left(t_{0}\right)\right),
\end{aligned}
$$

then

$$
\left|\bar{R}\left(f\left(t_{n}\right)\right)\right| \leq \frac{1}{\Gamma(2-\alpha)}\left[\frac{1-\alpha}{12}+\frac{2^{2-\alpha}}{2-\alpha}-\left(1+2^{-\alpha}\right)\right] \max _{0 \leq t \leq t_{n}}\left|f^{\prime \prime}(t)\right| \tau^{2-\alpha},
$$

where $0<\alpha \leq 1$ and $a_{j}=(j+1)^{1-\alpha}-j^{1-\alpha}, j \geq 0$.

Lemma 2.2 ([24]) Let $0<\alpha<1, a_{j}=(j+1)^{1-\alpha}-j^{1-\alpha}, j=0,1,2, \ldots$ Then

(1) $1=a_{0}>a_{1}>\cdots>a_{k} \rightarrow 0$, as $k \rightarrow+\infty$;

(2) $a_{n-1}>\frac{1-\alpha}{j^{2}}$.

Lemma 2.3 (Discrete Sobolev's inequality [19]) There exist constants $C_{1}$ and $C_{2}$ such that

$$
\left\|u^{n}\right\|_{\infty} \leq C_{1}\left\|u^{n}\right\|+C_{2}\left\|D_{+} u^{n}\right\|
$$

Lemma 2.4 (Discrete Gronwall's inequality [21]) Suppose that $\theta(k), \rho(k)$ are nonnegative mesh functions and $\rho(k)$ is nondecreasing. For $C>0$, if

$$
\theta(k) \leq \rho(k)+C \tau \sum_{l=0}^{k-1} \theta(l), \quad \forall k,
$$

then

$$
\theta(k) \leq \rho(k) e^{C \tau k}, \quad \forall k
$$

\section{Stability}

In this section, we study the stability of the proposed C-N-L1 scheme (6).

Theorem 3.1 The difference scheme (6) is unconditionally stable, and it holds

$$
\left\|u^{n}\right\|_{1} \leq C, \quad\left\|u^{n}\right\|_{\infty} \leq C, \quad n=0,1, \ldots, N .
$$

Proof For $n=0,1, \ldots, N$, computing the inner product of (6) with $2 u^{n+\frac{1}{2}}\left(i . e ., u^{n+1}+u^{n}\right)$, we obtain

$$
\begin{aligned}
& \frac{1}{\tau}\left(\left\|u^{n+1}\right\|^{2}-\left\|u^{n}\right\|^{2}\right)+\left(D_{0} u^{n+\frac{1}{2}}, 2 u^{n+\frac{1}{2}}\right)-\beta\left(D_{t} D^{2} u^{n}, 2 u^{n+\frac{1}{2}}\right) \\
& \quad+\gamma\left(Z, 2 u^{n+\frac{1}{2}}\right)-\mu\left(u^{n+\frac{1}{2}}-\sum_{i=0}^{n-1}\left(a_{i}-a_{i+1}\right) u_{j}^{n-\frac{1}{2}-i}-a_{n} u_{j}^{0}, 2 u^{n+\frac{1}{2}}\right) \\
& \quad-\alpha\left(D^{2} u^{n+\frac{1}{2}}, 2 u^{n+\frac{1}{2}}\right)=0,
\end{aligned}
$$

where $Z=\frac{1}{3}\left(u^{n} D_{0} u^{n+\frac{1}{2}}+D_{0}\left(u^{n} u^{n+\frac{1}{2}}\right)\right), \mu=\frac{\epsilon^{\frac{1}{2}} \tau^{-\frac{1}{2}}}{\Gamma\left(\frac{3}{2}\right)}$. 
For the second and fourth terms in (12), we use the definition of inner product and note that [20]

$$
\left(D_{0} u^{n+\frac{1}{2}}, 2 u^{n+\frac{1}{2}}\right)=\sum_{j=1}^{M}\left[u_{j+1}^{n+\frac{1}{2}} u_{j}^{n+\frac{1}{2}}-u_{j-1}^{n+\frac{1}{2}} u_{j}^{n+\frac{1}{2}}\right]=0,
$$

and

$$
\begin{aligned}
\left(Z, 2 u^{n+\frac{1}{2}}\right) & =\frac{2 h}{3} \sum_{j=1}^{M}\left(u_{j}^{n} D_{0} u_{j}^{n+\frac{1}{2}}+D_{0}\left(u^{n} u_{j}^{n+\frac{1}{2}}\right)\right) u_{j}^{n+\frac{1}{2}} \\
& =\frac{1}{3} \sum_{j=1}^{M}\left(u_{j}^{n}\left(u_{j+1}^{n+\frac{1}{2}}-u_{j-1}^{n+\frac{1}{2}}\right)+u_{j+1}^{n} u_{j+1}^{n+\frac{1}{2}}-u_{j-1}^{n} u_{j-1}^{n+\frac{1}{2}}\right) u_{j}^{n+\frac{1}{2}} \\
& =\frac{1}{3} \sum_{j=1}^{M}\left[\left(u_{j}^{n} u_{j+1}^{n+\frac{1}{2}} u_{j}^{n+\frac{1}{2}}+u_{j+1}^{n} u_{j+1}^{n+\frac{1}{2}} u_{j}^{n+\frac{1}{2}}\right)-\left(u_{j}^{n} u_{j-1}^{n+\frac{1}{2}} u_{j}^{n+\frac{1}{2}}+u_{j-1}^{n} u_{j-1}^{n+\frac{1}{2}} u_{j}^{n+\frac{1}{2}}\right)\right] \\
& =0 .
\end{aligned}
$$

From (13) and (14), (12) can be rewritten as

$$
\begin{aligned}
& \frac{1}{\tau}\left(\left\|u^{n+1}\right\|^{2}-\left\|u^{n}\right\|^{2}\right)+\frac{\beta}{\tau}\left(\left\|D_{+} u^{n+1}\right\|^{2}-\left\|D_{+} u^{n}\right\|^{2}\right)+2 \alpha\left\|D_{+} u^{n+\frac{1}{2}}\right\|^{2}+2 \mu\left\|u^{n+\frac{1}{2}}\right\|^{2} \\
& \quad=2 \mu \sum_{i=0}^{n-1}\left(a_{i}-a_{i+1}\right)\left(u^{n-\frac{1}{2}-i}, u^{n+\frac{1}{2}}\right)+2 \mu a_{n}\left(u^{0}, u^{n+\frac{1}{2}}\right) \\
& \quad \leq \mu \sum_{i=0}^{n-1}\left(a_{i}-a_{i+1}\right)\left(\left\|u^{n-\frac{1}{2}-i}\right\|^{2}+\left\|u^{n+\frac{1}{2}}\right\|^{2}\right)+\mu a_{n}\left(\left\|u^{0}\right\|^{2}+\left\|u^{n+\frac{1}{2}}\right\|^{2}\right) \\
& \quad=\mu \sum_{i=0}^{n-1}\left(a_{i}-a_{i+1}\right)\left\|u^{n-\frac{1}{2}-i}\right\|^{2}+\mu\left(\left(1-a_{n}\right)+a_{n}\right)\left\|u^{n+\frac{1}{2}}\right\|^{2}+\mu a_{n}\left\|u^{0}\right\|^{2} .
\end{aligned}
$$

Multiplying (15) by $\tau$, we have

$$
\begin{aligned}
& \left\|u^{n+1}\right\|_{1}^{2}+\tau \mu \sum_{i=0}^{n} a_{i+1}\left\|u^{n-\frac{1}{2}-i}\right\|^{2} \\
& \quad \leq\left\|u^{n}\right\|_{1}^{2}+\tau \mu \sum_{i=0}^{n-1} a_{i+1}\left\|u^{n-\frac{1}{2}-i}\right\|^{2}+\tau \mu a_{n}\left\|u^{0}\right\|^{2} .
\end{aligned}
$$

Let

$$
B^{n+1}=\left\|u^{n+1}\right\|_{1}^{2}+\tau \mu \sum_{i=0}^{n} a_{i+1}\left\|u^{n-\frac{1}{2}-i}\right\|^{2},
$$

(16) can be rewritten as follows:

$$
B^{n+1} \leq B^{n}+\mu a_{n}\left\|u^{0}\right\|^{2} .
$$


It follows that

$$
B^{n+1} \leq B^{0}+\mu \sqrt{n+1}\left\|u^{0}\right\|^{2} .
$$

Then we get

$$
\left\|u^{n}\right\|_{1} \leq C
$$

And using Lemma 2.3, we have

$$
\left\|u^{n}\right\|_{\infty} \leq C
$$

This completes the proof.

\section{Convergence}

In this section, the rate of convergence of scheme (6) is guaranteed and explicitly proved. Firstly, we consider the truncation error of the difference scheme (6). Let $v(x, t)$ be the solution of problems (2)-(4), $v_{j}^{n}=v\left(x_{j}, t_{n}\right)$, then the truncation error of scheme (6) is

$$
\begin{aligned}
r_{j}^{n}= & D_{t} v_{j}^{n}+D_{0} v_{j}^{n+\frac{1}{2}}-\beta D_{t} D^{2} v_{j}^{n}+\mu\left(v_{j}^{n+\frac{1}{2}}-\sum_{i=0}^{n-1}\left(a_{i}-a_{i+1}\right) v_{j}^{n-\frac{1}{2}-i}-a_{n} v_{j}^{0}\right) \\
& +\frac{\gamma}{3}\left(v_{j}^{n} D_{0} v_{j}^{n+\frac{1}{2}}+D_{0}\left(v_{j}^{n} v_{j}^{n+\frac{1}{2}}\right)\right)-\alpha D^{2} v_{j}^{n+\frac{1}{2}},
\end{aligned}
$$

where $\mu=\frac{\epsilon^{\frac{1}{2}} \tau^{-\frac{1}{2}}}{\Gamma\left(\frac{3}{2}\right)}$.

According to Taylor's expansion,

$$
\begin{aligned}
& D_{t} v_{j}^{n}+D_{0} v_{j}^{n+\frac{1}{2}}-\beta D_{t} D^{2} v_{j}^{n} \\
& +\left[\frac{\mu}{2}\left(\left(v_{j}^{n+1}+v_{j}^{n}\right)-\sum_{i=0}^{n-1}\left(a_{i}-a_{i+1}\right)\left(v_{j}^{n-i}+v_{j}^{n-1-i}\right)-a_{n} v_{j}^{0}\right)+O\left(\tau^{\frac{3}{2}}\right)\right] \\
& +\left\{\frac { \gamma } { 1 2 h } \left[u_{j}^{n}\left(u_{j+1}^{n+1}-u_{j-1}^{n+1}+u_{j+1}^{n}-u_{j-1}^{n}\right)\right.\right. \\
& \left.\left.+\left(u_{j+1}^{n} u_{j+1}^{n+1}-u_{j-1}^{n} u_{j-1}^{n+1}+u_{j+1}^{n} u_{j+1}^{n}-u_{j-1}^{n} u_{j-1}^{n}\right)\right]+O\left(\tau^{2}+h^{2}\right)\right\} \\
& \quad-\alpha D^{2} v_{j}^{n+\frac{1}{2}}=0,
\end{aligned}
$$

it can be easily obtained that $r_{j}^{n}=O\left(\tau^{\frac{3}{2}}+h^{2}\right)$ holds if $h, \tau \rightarrow 0$.

Theorem 4.1 Suppose $u_{0} \in H_{0}^{1}[0, L]$, then the solution $u_{j}^{n}$ of (6) converges to the solution $v_{j}^{n}$ of (2) in norm $\|\cdot\|_{\infty}$ and the rate of convergence is $O\left(\tau^{\frac{3}{2}}+h^{2}\right)$.

Proof Let $e_{j}^{n}=v_{j}^{n}-u_{j}^{n}$, subtracting (6) from (22), we have

$$
r_{j}^{n}=\frac{e_{j}^{n+1}-e_{j}^{n}}{\tau}+D_{0} e_{j}^{n+\frac{1}{2}}-\beta D_{t} D^{2} e_{j}^{n}
$$




$$
\begin{aligned}
& +\mu\left(e_{j}^{n+\frac{1}{2}}-\sum_{i=0}^{n-1}\left(a_{i}-a_{i+1}\right) e_{j}^{n-\frac{1}{2}-i}-a_{n} e_{j}^{0}\right) \\
& +\frac{\gamma}{3}\left(v_{j}^{n} D_{0} v_{j}^{n+\frac{1}{2}}-u_{j}^{n} D_{0} u_{j}^{n+\frac{1}{2}}\right) \\
& +\frac{\gamma}{3}\left(D_{0}\left(v_{j}^{n} v_{j}^{n+\frac{1}{2}}\right)-D_{0}\left(u_{j}^{n} u_{j}^{n+\frac{1}{2}}\right)\right)-\alpha D^{2} e_{j}^{n+\frac{1}{2}} .
\end{aligned}
$$

For convenience, we denote

$$
P=\frac{\gamma}{3}\left(v_{j}^{n} D_{0} v_{j}^{n+\frac{1}{2}}-u_{j}^{n} D_{0} u_{j}^{n+\frac{1}{2}}\right), \quad Q=\frac{\gamma}{3}\left(D_{0}\left(v_{j}^{n} v_{j}^{n+\frac{1}{2}}\right)-D_{0}\left(u_{j}^{n} u_{j}^{n+\frac{1}{2}}\right)\right)
$$

Multiplying by $2 e^{n+\frac{1}{2}}\left(i . e . e_{j}^{n+1}+e_{j}^{n}\right)$ on both sides of (24), we have

$$
\begin{aligned}
& \left\|e^{n+1}\right\|^{2}-\left\|e^{n}\right\|^{2}+\beta\left\|D_{+} e^{n+1}\right\|^{2}-\beta\left\|D_{+} e^{n}\right\|^{2} \\
& =\tau\left[-\left(D_{0} e^{n+\frac{1}{2}}, 2 e^{n+\frac{1}{2}}\right)-\left(P, 2 e^{n+\frac{1}{2}}\right)-\left(Q, 2 e^{n+\frac{1}{2}}\right)+\left(r^{n}, 2 e^{n+\frac{1}{2}}\right)\right. \\
& \quad-\mu\left(2\left\|e^{n+\frac{1}{2}}\right\|^{2}-\sum_{i=0}^{n-1}\left(a_{i}-a_{i+1}\right)\left(e^{n-\frac{1}{2}-i}, 2 e^{n+\frac{1}{2}}\right)-a_{n}\left(e^{0}, 2 e^{n+\frac{1}{2}}\right)\right) \\
& \left.\quad+\alpha\left(D^{2} e^{n+\frac{1}{2}}, 2 e^{n+\frac{1}{2}}\right)\right] .
\end{aligned}
$$

Now, we estimate the third and fourth terms of the right-hand side of (25), respectively $[18,20]$ :

$$
\begin{aligned}
\left(P, 2 e^{n+\frac{1}{2}}\right) & =\frac{2 h}{3} \sum_{j=1}^{M}\left(v_{j}^{n} D_{0} v_{j}^{n+\frac{1}{2}}-u_{j}^{n} D_{0} u_{j}^{n+\frac{1}{2}}\right) e_{j}^{n+\frac{1}{2}} \\
& =\frac{2 h}{3} \sum_{j=1}^{M}\left(v_{j}^{n} D_{0} e_{j}^{n+\frac{1}{2}}\right) e_{j}^{n+\frac{1}{2}}+\frac{2 h}{3} \sum_{j=1}^{M}\left(v_{j}^{n} D_{0} u_{j}^{n+\frac{1}{2}}-u_{j}^{n} D_{0} u_{j}^{n+\frac{1}{2}}\right) e_{j}^{n+\frac{1}{2}} \\
& \leq C h \sum_{j=1}^{M}\left(\left|D_{0} e_{j}^{n+\frac{1}{2}}\right|+\left|e_{j}^{n}\right|\right) e_{j}^{n+\frac{1}{2}} \\
& \leq C h\left(\left\|D_{+} e^{n+1}\right\|^{2}+\left\|D_{+} e^{n}\right\|^{2}+\left\|e^{n+1}\right\|^{2}+\left\|e^{n}\right\|^{2}\right)
\end{aligned}
$$

and

$$
\begin{aligned}
\left(Q, 2 e^{n+\frac{1}{2}}\right) & =\frac{2 h}{3} \sum_{j=1}^{M}\left[D_{0}\left(v_{j}^{n} v_{j}^{n+\frac{1}{2}}\right)-D_{0}\left(u_{j}^{n} u_{j}^{n+\frac{1}{2}}\right)\right] e_{j}^{n+\frac{1}{2}} \\
& =-\frac{2 h}{3} \sum_{j=1}^{M}\left(v_{j}^{n} e_{j}^{n+\frac{1}{2}}\right) D_{0} e_{j}^{n+\frac{1}{2}}+\frac{2 h}{3} \sum_{j=1}^{M}\left(v_{j}^{n}-u_{j}^{n}\right) u_{j}^{n+\frac{1}{2}} D_{0} e_{j}^{n+\frac{1}{2}} \\
& =-\frac{2 h}{3} \sum_{j=1}^{M}\left(v_{j}^{n} e_{j}^{n+\frac{1}{2}}\right) D_{0} e_{j}^{n+\frac{1}{2}}-\frac{2 h}{3} \sum_{j=1}^{M} e_{j}^{n} u_{j}^{n+\frac{1}{2}} D_{0} e_{j}^{n+\frac{1}{2}}
\end{aligned}
$$




$$
\begin{aligned}
& \leq C h \sum_{j=1}^{M}\left(\left|e_{j}^{n+\frac{1}{2}}\right|+\left|e_{j}^{n}\right|\right) D_{0} e_{j}^{n+\frac{1}{2}} \\
& \leq C h\left(\left\|D_{+} e^{n+1}\right\|^{2}+\left\|D_{+} e^{n}\right\|^{2}+\left\|e^{n+1}\right\|^{2}+\left\|e^{n}\right\|^{2}\right) .
\end{aligned}
$$

For the second term on the right-hand side of (25), we have

$$
\begin{aligned}
& \sum_{i=0}^{n-1}\left(a_{n-i-1}-a_{n-i}\right)\left(e^{i+\frac{1}{2}}, 2 e^{n+\frac{1}{2}}\right)+a_{n}\left(e^{0}, 2 e^{n+\frac{1}{2}}\right) \\
& \quad \leq \sum_{i=0}^{n-1}\left(a_{n-i-1}-a_{n-i}\right)\left(\left\|e^{i+\frac{1}{2}}\right\|^{2}+\left\|e^{n+\frac{1}{2}}\right\|^{2}\right)+a_{n}\left(\left\|e^{0}\right\|^{2}+\left\|e^{n+\frac{1}{2}}\right\|^{2}\right) \\
& \quad=\sum_{i=0}^{n-1}\left(a_{n-i-1}-a_{n-i}\right)\left\|e^{i+\frac{1}{2}}\right\|^{2}+\left(\left(1-a_{n}\right)+a_{n}\right)\left\|e^{n+\frac{1}{2}}\right\|^{2}+a_{n}\left\|e^{0}\right\|^{2} \\
& \quad=\sum_{i=0}^{n-1}\left(a_{n-i-1}-a_{n-i}\right)\left\|e^{i+\frac{1}{2}}\right\|^{2}+\left\|e^{n+\frac{1}{2}}\right\|^{2}+a_{n}\left\|e^{0}\right\|^{2} .
\end{aligned}
$$

Using Lemma 2.4, it is obvious that

$$
\alpha\left(D_{+} D_{-} e^{n+\frac{1}{2}}, 2 e^{n+\frac{1}{2}}\right)=-2 \alpha\left\|D_{+} e^{n+\frac{1}{2}}\right\|^{2}
$$

and

$$
\left(r^{n}, 2 e^{n+\frac{1}{2}}\right) \leq\left\|r^{n}\right\|^{2}+\frac{1}{2}\left(\left\|e^{n+1}\right\|^{2}+\left\|e^{n}\right\|^{2}\right)
$$

Substituting (26)-(30) into (25) and using the fact that $\left\|e^{0}\right\|=0$, we get

$$
\begin{aligned}
& \left\|e^{n+1}\right\|^{2}-\left\|e^{n}\right\|^{2}+\beta\left\|D_{+} e^{n+1}\right\|^{2}-\beta\left\|D_{+} e^{n}\right\|^{2}+2 \tau \mu\left\|e^{n+\frac{1}{2}}\right\|^{2} \\
& \leq \\
& \tau C\left(\left\|D_{+} e^{n+1}\right\|^{2}+\left\|D_{+} e^{n}\right\|^{2}+\left\|e^{n+1}\right\|^{2}+\left\|e^{n}\right\|^{2}\right) \\
& +\tau \mu\left[\sum_{i=0}^{n-1}\left(a_{n-i-1}-a_{n-i}\right)\left\|e^{i+\frac{1}{2}}\right\|^{2}+\left\|e^{n+\frac{1}{2}}\right\|^{2}\right] \\
& +\tau\left\|r^{n}\right\|^{2}+\frac{1}{2} \tau\left(\left\|e^{n+1}\right\|^{2}+\left\|e^{n}\right\|^{2}\right), \\
& \leq \tau C\left(\left\|D_{+} e^{n+1}\right\|^{2}+\left\|D_{+} e^{n}\right\|^{2}+\left\|e^{n+1}\right\|^{2}+\left\|e^{n}\right\|^{2}\right) \\
& +\tau \mu\left[\sum_{i=0}^{n-1}\left(\left\|e^{i}\right\|^{2}+\left\|e^{i+1}\right\|^{2}\right)+\left\|e^{n+\frac{1}{2}}\right\|^{2}\right] \\
& +\tau\left\|r^{n}\right\|^{2}+\frac{1}{2} \tau\left(\left\|e^{n+1}\right\|^{2}+\left\|e^{n}\right\|^{2}\right) .
\end{aligned}
$$

Using Lemma 2.4, we have

$$
(1-\tau C)\left(\left\|e^{n+1}\right\|_{1}^{2}-\left\|e^{n}\right\|_{1}^{2}\right) \leq 2 \tau C\left\|e^{n}\right\|_{1}^{2}+\tau\left\|r^{n}\right\|^{2}
$$


where $\left\|e^{n}\right\|_{1}^{2}=\left\|e^{n}\right\|^{2}+\beta\left\|D_{+} e^{n}\right\|^{2}$. Moreover, let $\tau$ be suitably small, then

$$
\left\|e^{n+1}\right\|_{1}^{2}-\left\|e^{n}\right\|_{1}^{2} \leq C \tau\left\|e^{n}\right\|_{1}^{2}+C \tau\left\|r^{n}\right\|^{2}
$$

Summing up (33) from 0 to $n$, we get

$$
\left\|e^{n+1}\right\|_{1}^{2} \leq\left\|e^{0}\right\|_{1}^{2}+C \tau \sum_{k=1}^{n}\left\|e^{k}\right\|_{1}^{2}+C \tau \sum_{k=1}^{n}\left\|r^{k}\right\|^{2} .
$$

Using Lemma 2.4 and the boundedness of $r^{k}$, we have

$$
\left\|e^{n+1}\right\|_{1}^{2} \leq O\left(\tau^{\frac{3}{2}}+h^{2}\right)^{2}
$$

By Lemma 2.3 and (35), we obtain

$$
\left\|e^{n}\right\| \leq O\left(\tau^{\frac{3}{2}}+h^{2}\right), \quad\left\|D_{+} e^{n}\right\| \leq O\left(\tau^{\frac{3}{2}}+h^{2}\right), \quad\left\|e^{n}\right\|_{\infty} \leq O\left(\tau^{\frac{3}{2}}+h^{2}\right) .
$$

This completes the proof of Theorem 4.1.

\section{Numerical experiments}

In this section, we provide two numerical experiments to show the performance of the proposed numerical scheme (6).

Example 1 We check the accuracy and convergence order of the numerical scheme (6). Since we do not obtain the exact solution of (2), we consider the nonhomogeneous problem

$$
\begin{aligned}
& u_{t}+u_{x}-\beta u_{x x t}+\epsilon_{0}^{\frac{1}{2}}{ }_{0}^{C} D_{t}^{\frac{1}{2}} u+\gamma u u_{x}-\alpha u_{x x}=s(x, t), \quad x \in(0,1), t \in(0,1], \\
& u(x, 0)=u_{0}(x), \quad x \in(0,1), \\
& u(0, t)=u(1, t)=0, \quad t \in(0,1],
\end{aligned}
$$

where the source term $s(x, t)$ is determined by the exact $u(x, t)=x^{4}(x-1) t^{\frac{3}{2}}$, which does not belong to $C^{2}[0, T]$.

In Tables 1-3, we list the error and convergence order for scheme (6) with the same parameters.

In Table 3, we can observe the convergence order is $\frac{3}{2}$ for $\epsilon=1$ and 2 for $\epsilon=0$, respectively. That is a good agreement with the theoretical prediction of Theorem 4.1.

Table 1 Error and convergence order in time when $h=1 / 1000, \epsilon=\alpha=\beta=\gamma=1$

\begin{tabular}{lllll}
\hline$\tau$ & $\|e\|$ & Order & $\|e\|_{\infty}$ & Order \\
\hline $1 / 10$ & $4.953935 \mathrm{e}-04$ & - & $9.350106 \mathrm{e}-04$ & - \\
$1 / 20$ & $1.595958 \mathrm{e}-04$ & 1.634152 & $3.029161 \mathrm{e}-04$ & 1.626064 \\
$1 / 40$ & $5.380815 \mathrm{e}-05$ & 1.568526 & $1.026424 \mathrm{e}-04$ & 1.561290 \\
$1 / 80$ & $1.847254 \mathrm{e}-05$ & 1.542441 & $3.545068 \mathrm{e}-05$ & 1.533742 \\
$1 / 160$ & $6.326795 \mathrm{e}-06$ & 1.545836 & $1.225924 \mathrm{e}-05$ & 1.531942 \\
\hline
\end{tabular}


Table 2 Error and convergence order in space when $\tau=1 / 1000, \epsilon=\alpha=\beta=\gamma=1$

\begin{tabular}{lllll}
\hline$h$ & $\|e\|$ & Order & $\|e\|_{\infty}$ & Order \\
\hline $1 / 10$ & $1.841489 \mathrm{e}-03$ & - & $2.653046 \mathrm{e}-03$ & - \\
$1 / 20$ & $5.047981 \mathrm{e}-04$ & 1.867094 & $7.279309 \mathrm{e}-04$ & 1.865776 \\
$1 / 40$ & $1.321991 \mathrm{e}-04$ & 1.932994 & $1.905037 \mathrm{e}-04$ & 1.933982 \\
$1 / 80$ & $3.365747 \mathrm{e}-05$ & 1.973713 & $4.854140 \mathrm{e}-05$ & 1.972531 \\
$1 / 160$ & $8.309556 \mathrm{e}-06$ & 2.018083 & $1.199653 \mathrm{e}-05$ & 2.016598 \\
\hline
\end{tabular}

Table 3 Error and convergence order in time and space when $\epsilon=0, \alpha=\beta=\gamma=1$

\begin{tabular}{lllll}
\hline$\tau=h$ & $\|e\|$ & Order & $\|e\|_{\infty}$ & Order \\
\hline $1 / 10$ & $1.588457 \mathrm{e}-03$ & - & $2.350020 \mathrm{e}-03$ & - \\
$1 / 20$ & $4.183324 \mathrm{e}-04$ & 1.924904 & $6.274669 \mathrm{e}-04$ & 1.905061 \\
$1 / 40$ & $1.030145 \mathrm{e}-04$ & 2.021802 & $1.585501 \mathrm{e}-04$ & 1.984600 \\
$1 / 80$ & $2.440490 \mathrm{e}-05$ & 2.077604 & $3.894461 \mathrm{e}-05$ & 2.025443 \\
$1 / 160$ & $5.878778 \mathrm{e}-06$ & 2.053582 & $9.517392 \mathrm{e}-06$ & 2.032785 \\
\hline
\end{tabular}

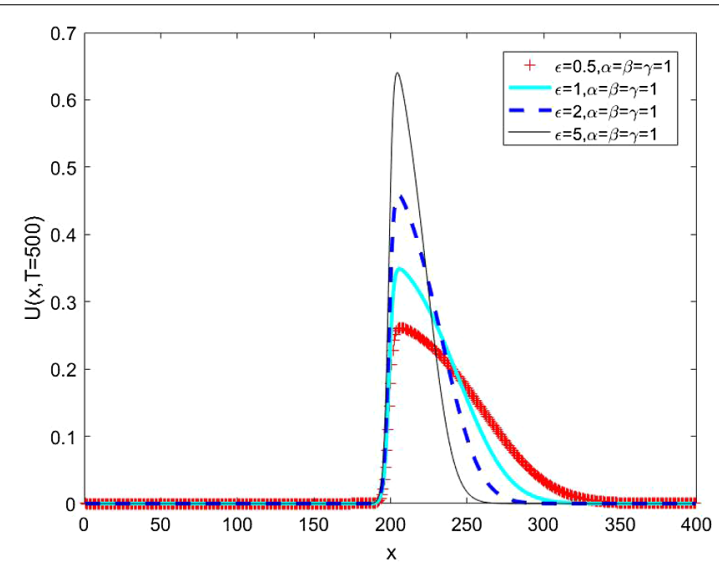

Figure $1 \epsilon$ ranging from 0.5 to 5 with fixed $\alpha=\beta=\gamma$

Example 2 We consider the following problem:

$$
\begin{aligned}
& u_{t}+u_{x}-\beta u_{x x t}+\epsilon_{0}^{\frac{1}{2} C} D_{t}^{\frac{1}{2}} u+\gamma u u_{x}-\alpha u_{x x}=0, \quad x \in(0,400), t \in(0, T], \\
& u(x, 0)=3 \operatorname{sech}^{2}\left(\sqrt{0.5^{3}}\left(x-x_{0}\right)\right),
\end{aligned}
$$

where $x_{0}$ is the middle point of the interval $(0,400)$ [10]. Since the initial value converges to 0 exponentially as $|x| \rightarrow+\infty$, we can approximate the initial value problem (38) by an initial-boundary value for $x \in(0,400)$ as long as the solution does not reach the boundaries. The numerical simulations are plotted in Figs. $1-3$.

In Fig. 1, we plot the solutions for several different values of $\epsilon$ with $\alpha, \beta$, and $\gamma$ fixed, and $M=500, \tau=1$. We observe that the nonlocal viscous term damps the wave down significantly.

Figure 2 shows the solutions for $\alpha, \beta, \gamma$ set to 0 or 1 with fixed $\epsilon$, and $M=500, \tau=1$. We can observe that the parameters $\alpha$ and $\beta$ have no significant impact on the wave evolution, whereas the parameter $\gamma$ plays an important role in wave shape.

Figure 3 shows the wave evolution in different time with $\alpha=\beta=\gamma=\epsilon=1$ and $\tau=1$. 


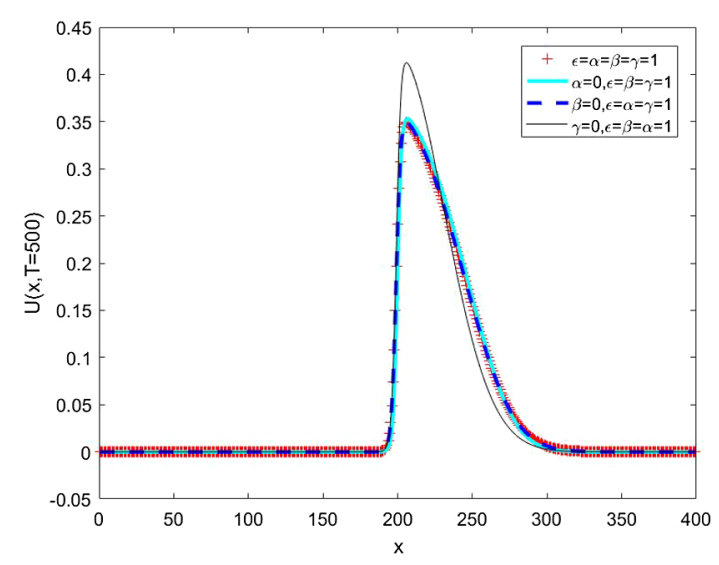

Figure $2 \alpha, \beta, \gamma$ set to 0 or 1 with fixed $\epsilon$

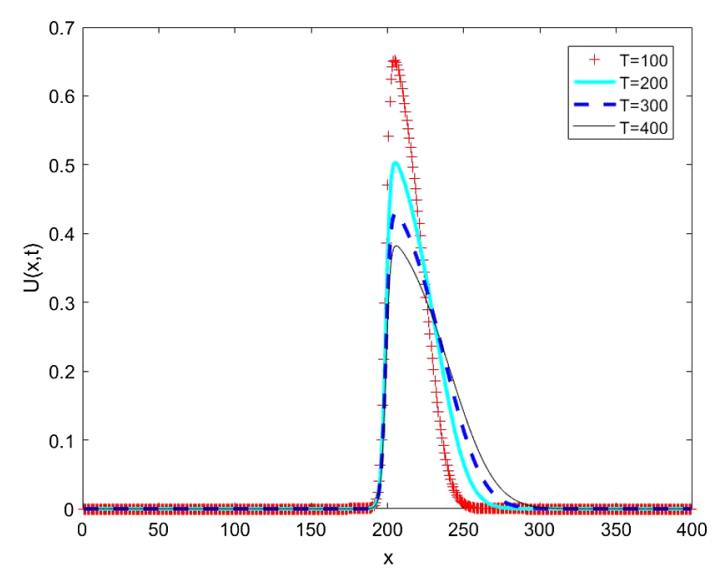

Figure 3 Time evolution of the solution for $\alpha=\beta=\gamma=\epsilon=1$

\section{Conclusions}

In this paper, we propose a $\mathrm{C}-\mathrm{N}-L 1$ linearized difference scheme for a nonlocal viscous wave equation in a BBM form. The convergence and stability of the scheme are discussed in detail. Numerical experiments show that our scheme is efficient and accurate. In addition, we also verify the influence of different parameters such as the diffusion term, nonlocal viscous term, dispersion term, and nonlinear term. We would extend the scope of our work in the future by investigating the finite element Galerkin scheme and compact finite difference scheme for the nonlocal viscous wave equation in a BBM form.

\section{Acknowledgements}

The authors would like to express their sincere thanks to the editor and the referees for the valuable comments and suggestions which helped to improve the original paper.

\section{Funding}

The work was supported by the fund of the Natural Science of Shandong Province (ZR2014AM033). 
Authors' contributions

The authors read and approved the final manuscript.

\section{Publisher's Note}

Springer Nature remains neutral with regard to jurisdictional claims in published maps and institutional affiliations.

Received: 2 June 2018 Accepted: 24 September 2018 Published online: 03 October 2018

\section{References}

1. Whitman, G.B., Fowler, R.G.: Linear and Nonlinear Waves. Wiley, New York (1974)

2. Bona, J.L., Smith, R.: The initial-value problem for the Korteweg-De Vries equation. Philos. Trans. R. Soc. Lond. B, Biol. Sci. 278(1287), 555-601 (1975)

3. Peregrine, D.H.: Long waves on a beach. J. Fluid Mech. 27(4), 815-827 (1967)

4. Peregrine, D.H.: Calculations of the development of an undular bore. J. Fluid Mech. 25, 321-330 (1966)

5. Rosenau, P.: A quasi-continuous description of a nonlinear transmission line. Phys. Scr. 34, 827-829 (1986)

6. Rosenau, P.: Dynamics of dense discrete systems. Prog. Theor. Phys. 79, 1028-1042 (1988)

7. Benjamin, T.B., Bona, J.J., Mahony, J.J.: Model equations for long waves in nonlinear dispersive systems. Philos. Trans. R. Soc. Lond. 272(1220), 47-78 (1972)

8. Wazwaz, A.M., Helal, M.A.: Nonlinear variants of the BBM equation with compact and noncompact physical structures. Chaos Solitons Fractals 26(3), 767-776 (2005)

9. Dutykh, D.: Numerical simulation of a solitonic gas in KdV and KdV-BBM equations. Phys. Lett. A 378(42), 3102-3110 (2014)

10. Zhang, J., Xu, C.: Finite difference/spectral approximations to a water wave model with a nonlocal viscous term. Appl. Math. Model. 38(19-20), 4912-4925 (2014)

11. Zhang, J., Li, W.L.: Numerical approximation to a shallow wave model with a nonlocal viscous. Chin. J. Eng. Math. 32(4), 577-589 (2015)

12. Chen, M., Dumont, S., Goubet, O.: Decay of solutions to a viscous asymptotical model for waterwaves: Kakutani-Matsuuchi model. Nonlinear Anal. 75(5), 2883-2896 (2012)

13. Li, C., Zhao, S.: Efficient numerical schemes for fractional water wave models. Comput. Math. Appl. 71(1), 238-254 (2016)

14. Bona, J.L., Pritchard, W.G., Scott, L.R.: Numerical schemes for a model for nonlinear dispersive waves. J. Comput. Phys. 60(2), 167-186 (1985)

15. $\mathrm{Hu}, \mathrm{B} ., \mathrm{Xu}, \mathrm{Y} ., \mathrm{Hu}, \mathrm{J}$.: Crank-Nicolson finite difference scheme for the Rosenau-Burgers equation. Appl. Math. Comput. 204(1), 311-316 (2008)

16. $\mathrm{Hu}, \mathrm{J} ., \mathrm{Hu}, \mathrm{B} . \mathrm{Xu}, \mathrm{Y}$.: Average implicit linear difference scheme for generalized Rosenau-Burgers equation. Appl. Math. Comput. 217(19), 7557-7563 (2011)

17. Janwised, J., Wongsaijai, B., Mouktonglang, T.: A modified three-level average linear-implicit finite difference method for the Rosenau-Burgers equation. Adv. Math. Phys. 2014(2), 139-150 (2014)

18. Xue, G.Y., Zhang, L.: A new finite difference scheme for generalized Rosenau-Burgers equation. Appl. Math. Comput. 222(5), 490-496 (2013)

19. Pan, X., Zhang, L.: Numerical simulation for general Rosenau-RLW equation: an average linearized conservative scheme. Math. Probl. Eng. 2012, 243-253 (2012)

20. Pan, X., Zhang, L.: A new finite difference scheme for the Rosenau-Burgers equation. Appl. Math. Comput. 2018(17), 8917-8924 (2012)

21. Li, C.: Linearized difference schemes for a BBM equation with a fractional nonlocal viscous term. Appl. Math. Comput. $311,240-250$ (2017)

22. Gorenflo, R., Mainardi, F.: Fractional calculus: integral and differential equations of fractional order. Math. 49(2), 277-290 (2008)

23. Langlands, T., Henry, B.: The accuracy and stability of an implicit solution method for the fractional diffusion equation. J. Comput. Phys. 205, 719-736 (2005)

24. Gao, G.H., Sun, Z.Z:: A compact finite difference scheme for the fractional sub-diffusion equations. J. Comput. Phys. 230, 586-595 (2011)

\section{Submit your manuscript to a SpringerOpen ${ }^{\circ}$ journal and benefit from:}

- Convenient online submission

- Rigorous peer review

- Open access: articles freely available online

- High visibility within the field

- Retaining the copyright to your article

Submit your next manuscript at $\boldsymbol{~ s p r i n g e r o p e n . c o m ~}$ 